\title{
The changing paradigm for supportive care in cancer patients
}

\author{
Alexandre Chan · Jude Lees • Dorothy Keefe
}

Received: 11 February 2014 / Accepted: 21 March 2014 / Published online: 10 April 2014

(C) Springer-Verlag Berlin Heidelberg 2014

Dear Editor,

With scientific advances in cancer supportive care and the incorporation of effective supportive care strategies into contemporary cancer treatment, there has been a substantial reduction in the occurrence of severe toxicities traditionally associated with cytotoxic chemotherapy over the past two decades. For example, appropriate prophylaxis with antiemetics (including a 5- $\mathrm{HT}_{3}$ antagonist, corticosteroid, and NK-1 antagonist) has greatly reduced chemotherapy-induced nausea and vomiting, colony-stimulating factors have curbed febrile neutropenia, and the integrated use of mouth care and palifermin has decreased the severity of oral mucositis. In addition, the introduction of structured management and care pathways into clinical practice has widely promoted these supportive care strategies for patients. We may not currently have all the answers on the prevention of chemotherapyinduced toxicities, but most oncology clinicians today can capably handle toxicities which were previously considered difficult to manage.

Innovations in cancer therapeutics have improved the oncologist's toolkit to cure and control many cancers previously difficult to manage. Targeted therapy agents are now

\footnotetext{
A. Chan

Department of Pharmacy, Faculty of Science, National University of Singapore, Singapore, Singapore

\section{A. Chan}

Department of Pharmacy, National Cancer Centre Singapore, Outram, Singapore

J. Lees $\cdot$ D. Keefe $(\bowtie)$

Royal Adelaide Hospital Cancer Centre, Royal Adelaide Hospital, North Terrace, Adelaide, SA 5000, Australia

e-mail: dorothy.keefe@health.sa.gov.au

D. Keefe

Faculty of Health Sciences, University of Adelaide, Adelaide, Australia
}

commonly used in the management of cancers ranging from chronic myeloid leukemia to renal cell carcinoma, and new indications and increased lines of therapy continue to arise. Initially "targeted" drugs were thought to have unique mechanisms for targeting cancer cells and were considered to produce fewer side effects than traditional cytotoxic chemotherapy. Importantly, however, clinicians are now observing a number of emerging and highly prevalent side effects associated with the use of targeted therapies [1]. These agents, which carry with them significant financial cost, are also associated with an increase in chronic complications that can decrease a patient's quality of life [2]. We must, therefore, strive to understand and be responsive to the side effects of these novel therapeutics to enable optimal patient adherence to and participation in their treatment.

In this commentary, we highlight toxicities that are commonly associated with targeted therapies, namely dermatological, gastrointestinal (diarrhea and stomatitis), hepatotoxicity, cardiotoxicity, neurotoxicity, and immunotoxicity. More importantly, we will emphasize the current impediments to understanding the mechanisms behind emerging side effects and the lack of effective strategies for managing these toxicities.

\section{Dermatological toxicities}

Commonly associated with targeted agents is a range of dermatological toxicities, which are different from the skin toxicities seen with traditional cytotoxic chemotherapy. The first of these class-related skin toxicities is observed with epidermal growth factor receptor inhibitors (EGFRI), including agents used for the treatment of lung (gefitinib, erlotinib, and afatinib), pancreatic (erlotinib), breast (lapatinib), head and neck (cetuximab), and colorectal cancers (cetuximab and panitumumab). They usually manifest as papulopustular rash (also referred to as acneiform rash), pruritus, xerosis/skin 
fissures, hair and nail changes, and mucositis. It is important to note that dermatological side effects of EGFRI are known to correlate with increased drug responses and the improvement of survival in several settings [3]. Despite the benefits, these side effects may result in significant physical and emotional discomfort to patients [4]; therefore, it is critical to maximize supportive care measures. The Multinational Association of Supportive Care in Cancer (MASCC), through its Skin Toxicities Study Group, has reviewed current literature and proposed important steps to address these dermatological issues. MASCC has published a guideline for the management of the skin toxicities associated with EGFRI [5]. However, it is important to note that quality studies that investigate the management of these dermatological toxicities are still sparse; hence, research in this area is urgently needed.

Other non-EGFRI-targeted therapy agents are associated with various dermatological toxicities. Vascular endothelial growth factor inhibitors such as sorafenib and sunitinib are commonly associated with hand foot syndrome (HFS), which is well documented to cause pain and reduce health-related quality of life $[6,7]$. Limited information is available to predict which patients are at risk of developing HFS, and effective management strategies are also lacking. Vemurafenib, which is a BRAF inhibitor, also causes rash, pruritus, and HFS. All grades of toxicity are observed in $19 \%$ of the patients receiving vemurafenib, with $2 \%$ experiencing high-grade toxicities [8]. Similar to the aforementioned targeted agents, patients experiencing severe dermatological toxicities from vemurafenib may require dose adjustment.

\section{Gastrointestinal toxicities}

Several types of gastrointestinal toxicities such as diarrhea and stomatitis are commonly associated with targeted therapies. Diarrhea is self-limiting, with all grades of diarrhea reported for approximately 50-60\% of patients treated with tyrosine kinase inhibitors (TKIs) and $30 \%$ of patients treated with mammalian target of rapamycin (mTOR) inhibitors [9]. It usually develops as early as 2 weeks into treatment. It has been suggested that there are three mechanisms of targeted therapy diarrhea-secretive diarrhea (with most of the EGFRI), direct ischemic mucosal damage and colitis (with VEGF inhibitors), and immunotoxicity related diarrhea (ipilimumab). The severity of diarrhea varies among patients and ranges from mild symptoms to fecal incontinence. Patients experiencing mild symptoms can find support in anti-diarrheal medications such as loperamide and diphenoxylate. Those experiencing severe symptoms may require dose interruptions and treatment adjustments.

Similar to diarrhea, stomatitis/mucositis is a major concern and usually occurs as early as 4-14 days after the initiation of targeted treatment. Several classes of agents including mTOR inhibitors (such as temsirolimus and everolimus) and EGFRI are known to cause stomatitis. In contrast to chemotherapyinduced stomatitis/mucositis with local inflammation and visible tissue damage, mucosal damage due to targeted therapies is less specific and differs according to types of targeted treatment. For instance, in contrast to ulceration associated with radiotherapy and chemotherapy, stomatitis observed with mTOR inhibitors presents with discrete aphthous-like ulcerations [10]. It is likely that impaired wound healing plays a role in the development of ulceration in patients using mTOR inhibitors. On the other hand, the proposed mechanism of EGFRI-induced stomatitis involves follicular occlusion by keratinocytes, leading to an inflammatory response. These toxicities may be exacerbated when the EGFRI is used in combination with radiotherapy and/or chemotherapy [5]. A clear understanding of the mechanisms that lead to stomatitis can provide insight into the prevention and therapeutic strategies necessary to improve patient care.

\section{Hepatotoxicity}

Hepatotoxicity is another emerging problem commonly observed with TKIs. The clinical presentation of hepatotoxic reactions caused by traditional cytotoxic chemotherapy occurs in a wide variety of patterns including ductular injury with cholestasis, parenchymal cell injury with steatosis, fibrosis or necrosis, vascular lesions such as hepatitis, peliosis, and venoocclusive disease. In contrast, the majority of patients with targeted agent-induced hepatotoxicity experience transaminitis (elevation of alanine and aspartate transaminase) with no significant effect on total bilirubin. This suggests that TKI hepatotoxicity is primarily hepatocellular and not cholestatic in nature. The precise mechanism of hepatotoxicity induced by targeted agents remains largely unknown. Some researchers have attributed it to the generation of reactive metabolites upon metabolism, which can interfere with cellular molecules, thereby affecting cell function and cell death. Immune-mediated mechanisms were also proposed. Currently, mandatory black box warnings were issued for five of the twenty-one approved TKIs, including lapatinib, sunitinib, pazopanib, regorafenib, and ponatinib. The frequencies of all grades of TKI hepatic adverse events vary from $11 \%$, as seen with gefitinib, to more than $50 \%$, with pazopanib. In terms of grade 3 and higher hepatic toxicity, frequencies range from 1 to $12 \%$ [11]. Clinicians must routinely monitor the liver function of patients receiving oral TKI, as there is limited information available to predict the occurrence of hepatic side effects. Specific strategies for management are specified in the product information. Further studies are required to investigate and further establish the mechanism of TKIassociated hepatotoxicity, in order to develop effective strategies to prevent its occurrence. 


\section{Cardiotoxicity}

Drug-induced cardiotoxicity is widely considered a potentially fatal complication within the realm of cancer supportive care. The most common clinical presentation is dose-dependent cardiomyopathy leading to congestive heart failure, which occurs after the administration of cytotoxic chemotherapy including anthracyclines. Throughout the past few decades, we have learned how to prevent and manage this cardiotoxicity. Patients are at risk of various types of cardiotoxicity in the targeted therapy era, including left ventricular dysfunction, hypertension, and QT prolongation. A number of targeted agents including trastuzumab, imatinib, and sunitinib are known to induce cardiomyopathy. Trastuzumab, which is a humanized monoclonal antibody designed to target HER2 on the surface of HER2overexpressing tumor cells, is associated with a 2 to $28 \%$ incidence rate of left ventricular dysfunction [12]. Several patient-related risk factors were identified to increase the risk of trastuzumab-induced cardiomyopathy, including older age, history of cardiovascular disease, and prior treatment with anthracyclines. Although the exact mechanism of trastuzumab-induced cardiac dysfunction is not well understood, the currently available literature suggests that it may differ from anthracyclines. For example, trastuzumab-induced cardiomyopathy has a high tendency for reversal after the temporary cessation of therapy. Unlike the anthracyclines, there is no evidence that cumulative doses can predict for cardiomyopathy with trastuzumab. Guidelines advise that patients suffering heart failure due to anticancer agents are managed similarly to those with heart failure due to other causes [13]. However, it is unclear whether cancer patients respond similarly to non-cancer patients. Research has recently focused on the role of biomarkers such as troponin and B-type natriuretic peptide in the occurrence of cardiomyopathy [14].

Another type of cardiotoxicity that has emerged with targeted therapy is hypertension. Angiogenesis inhibitors such as bevacizumab, sunitinib, sorafenib, and pazopanib are known to induce hypertension [15]. The mechanism involves the inhibition of VEGF on vasculatures, leading to decreases in nitric oxide and prostaglandin I2 and subsequently to increased vasoconstriction and blood pressure. The ideal agent for the management of hypertension caused by angiogenesis is currently unknown. However, manufacturers of the various agents recommend routine blood pressure monitoring and standard anti-hypertensive therapy. Blood pressure should also be well controlled prior to initiating these agents. Furthermore, a number of targeted therapies also put some patients at risk of QT prolongation. Nilotinib, which is a second-generation TKI used in chronic myelogenous leukemia, can cause QT prolongation and sudden cardiac death. Hence, patients receiving nilotinib should undergo ECG monitoring at baseline, 7 days after initiation, and periodically thereafter. We should ensure that patients avoid taking medications that prolong the QT interval and drugs that are strong CYP3A4 inhibitors.

\section{Pulmonary toxicity}

Interstitial lung disease (ILD), a noninfectious cause of pneumonitis, is observed more often with the use of targeted therapies and more commonly known to be associated with the use of EGFRI or mTOR inhibitors. With EGFRI, the incidence is relatively rare with the exception of Japanese patients [16]. In contrast, the occurrence of mTOR inhibitorinduced ILD is far more common, especially agents such as everolimus are increasingly used in the management of advanced breast cancer and metastatic renal cell carcinoma [17]. Onset of the pulmonary toxicity occurs relatively early, and most of these patients would present with cough and dyspnea. Radiographic findings may find patchy diffuse ground-glass opacities. In patients who develop severe symptoms associated with ILD, it is recommended to stop therapy, manage symptoms with corticosteroids, and rule out infectiousinduced pneumonia with pulmonary evaluation.

\section{Neurotoxicity}

Although the literature has thoroughly described the mechanisms of chemotherapy-induced peripheral neuropathy (such as seen with taxanes and vinca alkaloids), the mechanism for targeted therapy-related peripheral neuropathy is still under investigation. Proteasome inhibitors such as bortezomib cause neurotoxicity which is generally reversible. Most patients are managed by dose reduction or by changing the route of administration. Interestingly, carfizomib, a newly available proteasome inhibitor, does not cause neurotoxicity, suggesting that the associated toxicity is not a class effect. It is important for us to know the mechanism behind this side effect to develop effective pharmacological strategies for the treatment of bortezomib-induced peripheral neuropathy.

Emerging evidence has suggested that targeted therapies may also lead to cognitive impairment. VEGF has a possible role in brain cognition, which gives rise to concerns that VEGF inhibitors may induce neurotoxic effects in cancer patients. Furthermore, VEGF may affect cognition in various ways via its roles in angiogenesis, neurogenesis, and neuronal plasticity, and the use of VEGF inhibitors in cancer treatment may cause cognitive impairment. To date, clinical data suggest that patients who received sorafenib or sunitinib experienced a significant cognitive impairment in the domains of memory and learning, executive functioning, and abstract reasoning relative to a healthy control group. However, conflicting evidence also suggests that anti-angiogenic therapies may not have any effects on cognitive function. These conflicting results make it difficult to ascertain the relationship between treatment-related 
cognitive changes and the use of VEGF inhibitors [18]. This inability to observe a cognitive impairment may be attributable to a poor study design, such as the use of tools that were not optimized or validated, and a small sample size.

\section{Immunotoxicity}

Ipilimumab is a monoclonal antibody that targets cytotoxic $\mathrm{T}$ lymphocyte antigen 4 (CTLA-4) and is known to induce immunotoxicities, which usually present as dermatitis (pruritus, rash), enterocolitis, endocrinopathies, liver abnormalities, uveitis, neurotoxicities, and hematopoietic toxicities. The onset of immunotoxicities can be quite sudden; severity can range from mild to life threatening, but they uncommonly result in significant morbidity or death. The mechanisms of immunotoxicities appear to be T-cell mediated, which is characterized by T-cell-rich inflammatory infiltrates with some suggestion of circulating antibodies. Immunotoxicities may follow an unpredictable course, and if untreated can be severe and life threatening. The primary treatment for most lowgrade immunotoxicities is supportive care; high-dose steroids and withholding further ipilimumab are recommended for higher grade immunotoxicities. Permanent cessation of antiCTLA-4 therapy is recommended in severe cases, but the majority of patients respond to corticosteroid therapy [19]. As such toxicities are insidious in nature, it is important to develop strategies for understanding how they arise.

How should we move forward?

We have briefly highlighted a number of areas in which supportive care for cancer patients who have been prescribed targeted therapies is an emerging challenge. This challenge is exacerbated by the expected increase in the number and type of targeted therapies that will enter the oncologist's arsenal based on new research and approvals. As the science concerning the toxicities associated with targeted therapies remains unconfirmed, we still need to manage our patients. We must go beyond our comfort zone and research the epidemiology, pathobiology, and treatment strategies for these new agents. New algorithms are required, and we must explore the management of these toxicities using novel approaches, including pharmacogenomics and genetic risk prediction in order to personalize management strategies [20]. On a larger platform, as a leading organization in cancer supportive care, MASCC should charge its various study groups to take an active lead in advancing the research and clinical management of these emerging toxicities. A multidisciplinary approach is required to ensure the optimal treatment outcome for our patients receiving targeted treatment. It is with excitement and enthusiasm as we explore this new frontier of research to deliver a more effective cancer care for our patients. It opens up opportunities for MASCC to explore new directions and potentially a new structure in response to the tremendous changes taking place as the science advances. We owe it to our patients to investigate our potential to be responsive and effective.

\section{Conflict of interest None}

\section{References}

1. Keefe DM, Bateman EH (2012) Tumor control versus adverse events with targeted anticancer therapies. Nat Rev Clin Oncol 9:98-109

2. Chan A, Chiang YY, Low XH et al (2013) Affordability of cancer treatment for aging cancer patients in Singapore: an analysis of health, lifestyle, and financial burden. Support Care Cancer 21: 3509-3517

3. Liu HB, Wu Y, Lv TF et al (2013) Skin rash could predict the response to EGFR tyrosine kinase inhibitor and the prognosis for patients with non-small cell lung cancer: a systematic review and meta-analysis. PLoS One 8:e55128

4. Boers-Doets CB, Gelderblom H, Lacouture ME et al (2013) Experiences with the FACT-EGFRI-18 instrument in EGFRIassociated mucocutaneous adverse events. Support Care Cancer 21: 1919-1926

5. Lacouture ME, Anadkat MJ, Bensadoun RJ et al (2011) Clinical practice guidelines for the prevention and treatment of EGFR inhibitor-associated dermatologic toxicities. Support Care Cancer 19:1079-1095

6. Nardone B, Hensley JR, Kulik L et al (2012) The effect of hand-foot skin reaction associated with the multikinase inhibitors sorafenib and sunitinib on health-related quality of life. J Drugs Dermatol 11:e61e65

7. Teo YL, Chong XJ, Chue XP et al (2014) Role of sunitinib and SU12662 on dermatological toxicities in metastatic renal cell carcinoma patients: in vitro, in vivo, and outcomes investigation. Cancer Chemother Pharmacol 73:381-388

8. Huang V, Hepper D, Anadkat M, Cornelius L (2012) Cutaneous toxic effects associated with vemurafenib and inhibition of the BRAF pathway. Arch Dermatol 148:628-633

9. Pessi MA, Zilembo N, Haspinger ER et al. Targeted therapy-induced diarrhea: a review of the literature. Crit Rev Oncol Hematol 2013.

10. Boers-Doets CB, Raber-Durlacher JE, Treister NS et al (2013) Mammalian target of rapamycin inhibitor-associated stomatitis. Future Oncol 9:1883-1892

11. Teo YL, Ho HK, Chan A (2013) Risk of tyrosine kinase inhibitorsinduced hepatotoxicity in cancer patients: a meta-analysis. Cancer Treat Rev 39:199-206

12. Yeh ET, Bickford CL (2009) Cardiovascular complications of cancer therapy: incidence, pathogenesis, diagnosis, and management. J Am Coll Cardiol 53:2231-2247

13. Guarneri V, Lenihan DJ, Valero V et al (2006) Long-term cardiac tolerability of trastuzumab in metastatic breast cancer: the M.D. Anderson Cancer Center experience. J Clin Oncol 24:4107-4115

14. Cardinale D, Colombo A, Torrisi R et al (2010) Trastuzumab-induced cardiotoxicity: clinical and prognostic implications of troponin I evaluation. J Clin Oncol 28:3910-3916

15. Keefe D, Bowen J, Gibson R et al (2011) Noncardiac vascular toxicities of vascular endothelial growth factor inhibitors in advanced cancer: a review. Oncologist 16:432-444

16. Kudoh S, Kato H, Nishiwaki Y et al (2008) Interstitial lung disease in Japanese patients with lung cancer: a cohort and nested case-control study. Am J Respir Crit Care Med 177:1348-1357 
17. Albiges L, Chamming's F, Duclos B et al (2012) Incidence and management of mTOR inhibitor-associated pneumonitis in patients with metastatic renal cell carcinoma. Ann Oncol 23:1943-1953

18. Ng T, Cheung YT, Ng QS et al (2014) Vascular endothelial growth factor inhibitors and cognitive impairment: evidence and controversies. Expert Opin Drug Saf 13:83-92
19. Fecher LA, Agarwala SS, Hodi FS, Weber JS (2013) Ipilimumab and its toxicities: a multidisciplinary approach. Oncologist 18:733-743

20. Liu G, Cheng D, Ding K et al (2012) Pharmacogenetic analysis of BR.21, a placebo-controlled randomized phase III clinical trial of erlotinib in advanced non-small cell lung cancer. J Thorac Oncol 7: $316-322$ 